\title{
How to Measure Our Sensitivity in Order to Better Understand What Happens and Decide What to Do
}

\author{
Diego Liberati* \\ Consiglio Nazionale delle Ricerche, Italy
}

*Corresponding author: Diego Liberati, Consiglio Nazionale delle Ricerche, Italy.

Received Date: August 12, 2018

Published Date: August 28, 2018

\section{Editorial}

We are obviously sensitive to every kind of stressors. To measure effects is of paramount necessity. A first not specific but quite sensitive way to detect and quantify stress is heart rate variability: sympatho-vagal balance is known to be altered either in acute or even chronically according to the state and of the dynamics of each of us; specificity could be at least partially achieved by keeping fixed as much as possible every other stimuli [1]. Less immediate, and still originally a-specific, is the analysis of the central nervous system instead of the autonomous one: coherences among brain areas, investigated via EEG, MEG, fMRI, NIRS, do even account on our plasticity to the change [2]. Deconvolution of blood samples may help in noninvasively assessing un-accessible and nano-metric pituitary secretion in controlling hormone loops [3]. A real precision analysis is needed to achieve very specific results: epigenetics makes us enhancing gene mediated protein expression in such a way that salient involved genes are detectable in assays together with their networking behavior with proteins expressed to face stimuli [4]. Modeling biophysical and biochemical interactions at molecular, domain and even atomic scale could become the ultimate level in approaching the effect from macro to meso, coarse and micro scales $[5,6]$.

\section{References}

1. Pagani M, Mazzuero G, Ferrari A, Liberati D, Cerutti S et al. (1991) Sympathovagal interaction during mental stress: a study employing spectral analysis of heart rate variability in healthy controls and in patients with a prior myocardial infarction. Circulation 83(4) II: 43-51.

2. Baraldi P, Manginelli AA, Maieron M, Liberati D, Porro CA (2007) An ARX model-based approach to trial by trial identification of fMRI-BOLD responses. NeuroImage 37(1): 189-201.

3. De Nicolao G, Liberati D (1993) Linear and Nonlinear Techniques for the Deconvolution of Hormone Time-Series. IEEE Transactions on Biomedical Engineering 40 (5): 440-455.

4. Muselli M, Liberati D (2002) Binary rule generation via Hamming Clustering. IEEE Transactions on Knowledge and Data Engineering 14(6): 1258-1268.

5. Mezza D, Pappalettera M, Liberati D (2016) A quantitative numerical model for TNF- $\alpha$ mediated cellular Apoptosis. Biol Eng Med 1(1): 1-8.

6. Sacco E, Farina M, Greco C, Lamperti S, Busti S, et al. (2012) Regulation of hSos 1 activity is a system-level property generated by its multi-domain structure. Biotechnol Adv 30(1): 154-168. 